JOURNAL OF

SYNCHROTRON

RADIATION

ISSN 1600-5775

Received 16 February 2017

Accepted 18 June 2017

Edited by S. Svensson, Uppsala University, Sweden

Keywords: single-photon counters; hybrid pixel detectors; instrumentation for synchrotron radiation accelerators; X-ray detectors; PEEM; LEEM.

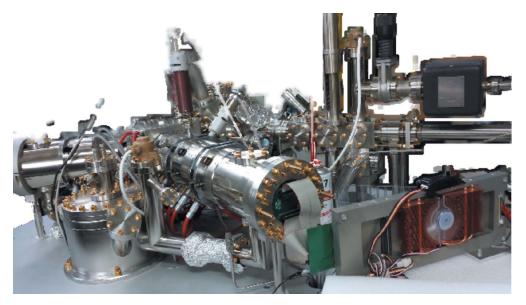

(C) 2017 International Union of Crystallography

\section{The EIGER detector for low-energy electron microscopy and photoemission electron microscopy}

\author{
G. Tinti, ${ }^{a *}$ H. Marchetto, ${ }^{\text {b,c }}$ C. A. F. Vaz, ${ }^{a}$ A. Kleibert, ${ }^{a}$ M. Andrä, ${ }^{a}$ R. Barten, ${ }^{a}$ \\ A. Bergamaschi, ${ }^{a}$ M. Brückner, ${ }^{\text {a }}$ S. Cartier, ${ }^{a}$ R. Dinapoli, ${ }^{a}$ T. Franz, ${ }^{\text {b }}$ E. Fröjdh, ${ }^{\text {a }}$ \\ D. Greiffenberg, ${ }^{a}$ C. Lopez-Cuenca, ${ }^{a}$ D. Mezza, ${ }^{a}$ A. Mozzanica, ${ }^{a}$ F. Nolting, ${ }^{a}$ \\ M. Ramilli, ${ }^{a}$ S. Redford, ${ }^{\text {a }}$ M. Ruat, ${ }^{\text {a Ch. Ruder, }}{ }^{\text {a }}$ L. Schädler, ${ }^{\text {a }}$ Th. Schmidt, ${ }^{\text {c }}$ \\ B. Schmitt, ${ }^{a}$ F. Schütz, ${ }^{b}$ X. Shi, ${ }^{a}$ D. Thattil, ${ }^{a}$ S. Vetter ${ }^{a}$ and J. Zhang ${ }^{a}$
}

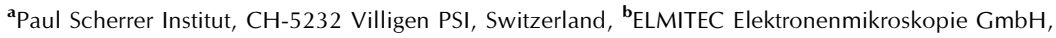
D-38678 Clausthal-Zellerfeld, Germany, and ${ }^{\mathrm{C}}$ Fritz-Haber-Institute of the Max-Planck-Society, Department of Chemical Physics, D-14195 Berlin, Germany. *Correspondence e-mail: gemma.tinti@psi.ch

EIGER is a single-photon-counting hybrid pixel detector developed at the Paul Scherrer Institut, Switzerland. It is designed for applications at synchrotron light sources with photon energies above $5 \mathrm{keV}$. Features of EIGER include a small pixel size $(75 \mu \mathrm{m} \times 75 \mu \mathrm{m})$, a high frame rate (up to $23 \mathrm{kHz}$ ), a small dead-time between frames (down to $3 \mu \mathrm{s}$ ) and a dynamic range up to 32-bit. In this article, the use of EIGER as a detector for electrons in low-energy electron microscopy (LEEM) and photoemission electron microscopy (PEEM) is reported. It is demonstrated that, with only a minimal modification to the sensitive part of the detector, EIGER is able to detect electrons emitted or reflected by the sample and accelerated to $8-20 \mathrm{keV}$. The imaging capabilities are shown to be superior to the standard microchannel plate detector for these types of applications. This is due to the much higher signal-to-noise ratio, better homogeneity and improved dynamic range. In addition, the operation of the EIGER detector is not affected by radiation damage from electrons in the present energy range and guarantees more stable performance over time. To benchmark the detector capabilities, LEEM experiments are performed on selected surfaces and the magnetic and electronic properties of individual iron nanoparticles with sizes ranging from 8 to $22 \mathrm{~nm}$ are detected using the PEEM endstation at the Surface/ Interface Microscopy (SIM) beamline of the Swiss Light Source.

\section{Introduction}

Low-energy electron microscopy (LEEM) and photoemission electron microscopy (PEEM) instruments are highly versatile characterization tools that are widely used in a vast range of research fields, from nanomagnetism and surface science to catalysis and battery research (Locatelli \& Bauer, 2008; Rodríguez et al., 2010; Cheng \& Keavney, 2012; Karim et al., 2017; Leanza et al., 2017). LEEM/PEEM relies on directly imaging photoelectrons locally emitted from the sample surface induced by illumination with an excitation source, such as electrons, UV-light or X-rays (Kuch et al., 2001; Bauer, 2014; Anders et al., 1999; Schneider, 1999). It explores the extreme sensitivity of the photoemitted electron intensity to local variations in the workfunction of the sample surface or to the nature of the excitation source which, combined with the high spatial resolution attainable, can be used to investigate surface science phenomena in LEEM and/or the spectroscopic response of materials in PEEM in real space, down to the nanometre scale. These unique features, together with recent technical advances including high lateral and energy resolu- 
tions (Schmidt et al., 2010, 2013), sample cooling stages and the availability of dedicated sample environments allowing the application of local magnetic, electrical and laser light pulses, have led to an increased availability of LEEM/PEEM instruments at synchrotron research facilities worldwide as well as in numerous research laboratories. A common feature of these instruments is that, in all possible operation modes, electrons are detected. An adjustable electron optics typically allows one to combine real-space investigations with resolution in the nanometre range with local $k$-space investigations in situ and on a very small sample area. To fully benefit from these techniques, dedicated electron detectors are required. However, since the invention of LEEM/PEEM (Griffith \& Engel, 1991; Engel et al., 1991; Veneklasen, 1991), the vast majority of these systems rely on detectors consisting of a double-chevron microchannel plate (MCP) operated with a bias voltage typically between 1.0 and $1.6 \mathrm{kV}$, a phosphor screen at $5.5 \mathrm{kV}$ and a CCD camera. Advantages of MCPs include the fact that they are ultrahigh-vacuum (UHV) compatible, they can be heated well above $150^{\circ} \mathrm{C}$ for bakeout, and can be used for real-time imaging. There are, however, several drawbacks that limit the overall performance of these detectors, including high noise levels, low dynamic range, strong lateral gain inhomogeneities (partially compensated in post-processing normalization), low lateral resolution (Moldovan et al., 2008), irreversible local gain reductions upon exposure to high fluxes, and mechanical brittleness. Moreover, state-of-the-art experiments increasingly require the detection of smaller signals, such as in pump-probe experiments, or of a small signal overlaid on a very intense background, as in nanostructures. Also, the emergence of imaging aberration correctors comes intrinsically with enhanced transmission (Schmidt et al., 2013) and higher signal levels. Therefore, the development of new detectors simultaneously capable of high dynamic range and high signal-to-noise sensitivity is essential for the success of future experiments exploring ultrafast phenomena and nanoscale physical processes.

The recent progress in electron detectors, in particular in pixelated detectors with direct single-electron counting capabilities, have led recently to a new paradigm in transmission electron microscopy by improving the detection efficiency by orders of magnitude (Kühlbrandt, 2014; McMullan et al., 2009). Indeed, the excellent results from McMullan et al. (2007) and Nederlof et al. (2013) have driven the very recent developments and the commercialization of hybrid pixel detectors in transmission electron microscopes (Quantum Detectors, 2017; Dectris, 2017; Amsterdam Scientific Instruments, 2017) and scanning transmission electron microscopes (Krajnak et al., 2016; Raighne et al., 2011) at high kinetic energies (40-300 keV) and less stringent vacuum constraints. Also, single-photon-counting pixel detectors like PILATUS (Kraft et al., 2009), EIGER (Dinapoli et al., 2011) and Medipix (Llopart et al., 2002; Gimenez et al., 2015) are widely used in photon science and synchrotron experiments. The EIGER detector has been developed at the Paul Scherrer Institut (PSI), Switzerland, with the specific aim of satisfying the detector requirements for synchrotron applications, having a very high frame rate combined with small pixel size. The performance of EIGER as a single-photon-counting detector has already been reported (Johnson et al., 2014; Tinti et al., 2015) and various EIGER detector systems are in use at the Swiss Light Source (SLS) at PSI for diffraction experiments such as protein crystallography (Casanas et al., 2016), coherent diffraction imaging and ptychography (Guizar-Sicairos et al., 2014).

Here we report on LEEM/PEEM performance improvements thanks to the EIGER single-particle-counting detector at electron energies in the range $8-20 \mathrm{keV}$. By calibrating the pixel detector in absolute particle energy, we show that the full energy of the electron cannot be detected in a standard X-ray Si sensor, since a fraction of the electron energy is lost due to the attenuation at the entrance window of the sensor and due to multiple scattering. In addition, we present the design of a set-up that is compatible with high-vacuum constraints and optimized for low-energy electron measurements. For lowenergy electrons $(<20 \mathrm{keV})$ similar attempts to the one presented here have been performed by the Medipix2 collaboration (van Gastel et al., 2009; Sikharulidze et al., 2011) and proved the suitability of hybrid pixel detectors for low-energy electron detection. However, they have not resulted in a device specifically optimized for low electron energy detection to be permanently installed in an electron microscope for PEEM, which is the aim of this paper.

This paper is organized as follows. The description of the detector, integration into the ELMITEC microscope and its characterization using photons and electrons are presented in $\S 2$. In $\$ 3$ and $\$ 4$ we carry out the detector characterization necessary to determine the optimal conditions for performing imaging in an electron microscope. The study of electric and magnetic properties of $\mathrm{Fe}$ nanoparticles carried out at the Surface/Interface Microscopy (SIM) beamline at the SLS using the EIGER detector is described in §5. A comparison with the image quality obtained using the standard set-up for ELMITEC PEEM microscopes is also given. In $\S 6$ we describe future work towards an optimized and fully integrated EIGER detector for PEEM.

\section{The experimental set-up}

\subsection{The EIGER detector}

EIGER is a hybrid pixel detector, meaning that every pixel on the sensor, i.e. the radiation-sensitive part, is connected to a pixel on the readout chip, which performs the first electronic processing of the signal. The pixel size in EIGER is $75 \mu \mathrm{m} \times$ $75 \mu \mathrm{m}$ and the Si sensor used in this experiment is $320 \mu \mathrm{m}$ thick, manufactured by HAMAMATSU. The pixels are $\mathrm{p}^{+}$ implants on n-type Si substrate. For more details on pixel sensors, the reader is referred to Rossi et al. (2006). The entrance window, which is the first part of the sensor seen by the radiation, consists of $\sim 1 \mu \mathrm{m} \mathrm{Al}$ for applying the sensor bias voltage and a $\sim 2 \mu \mathrm{m}$-thick $\mathrm{n}^{+}$implant (non-depleted) $\mathrm{Si}$ layer. Both the $\mathrm{Al}$ and this $\mathrm{Si}$ backplane layer are material layers that a particle has to cross before being able to interact 
in the detecting area of the depleted Si bulk. The sensor is biased at $150 \mathrm{~V}$ in the experiments presented here. The signal charge produced by the detected particle will drift under the applied electric field to the pixel implants. The readout chip is designed in UMC $0.25 \mu \mathrm{m}$ technology. Radiation-hardening techniques have been used to extend the radiation hardness up to $0.3 \mathrm{kGy}$. The pixel matrix is composed of $256 \times 256$ pixels, for an overall dimension of a single chip of approximately $2 \mathrm{~cm} \times 2 \mathrm{~cm}$. Each pixel cell performs amplification of the signal from the sensor, with a user-configurable gain set by changing the amplifier feedback resistor. The user-configurable gain gives sensitivity to different signal pulse heights and, thus, to different particle energies. The signal, after being shaped, is then compared with the level at which the threshold has been set. If the signal is above the threshold, the counter in the pixel cell is incremented by one. The counter can be configured in 4-, 8- or 12-bit mode, allowing for a trade-off between high frame rate $\left(23,12\right.$ and $8 \mathrm{kHz}$, respectively) ${ }^{\mathbf{1}}$ and higher dynamic range (limited by the counter depth). In the experiments shown here, we acquired data at up to a maximum dynamic range of $4 \times 10^{3}$ (given by the depth of the 12-bit counter). EIGER offers the option of starting a new acquisition while reading the counters of the previous acquisition, giving a dead-time between frames of as little as $3 \mu \mathrm{s}$.

In the experiments presented here, a single chip is used. However, since the chips are three-side buttable, more chips can be bonded to a larger single sensor, allowing for detection of a wider area with no efficiency loss between the chips thanks to correspondingly larger pixels $(\times 2$ along the sides, $\times 4$ in the corners) in the sensor at the chip boundaries. The counter values are read out by an electronic board, which can handle data from a maximum of four readout chips. They perform digital data processing through field programmable gate arrays (FPGAs) and store data on DDR2 memories. Onboard image summation is used to extend the dynamic range from 12-bit to 32-bit, providing a counter value up to $4 \times 10^{9}$ and reducing the load of the data transfer from the board to the PC. When setting the detector in 32-bit mode (i.e. autosumming mode), a single longer acquisition is broken down into a series of short (typically $2 \mathrm{~ms}$ ) 12-bit acquisitions, separated by a dead-time of $10 \mu \mathrm{s}$.

In this study, we took data at a frame rate slower than $1 \mathrm{~Hz}$ due to the long exposure times needed for good statistics when performing calibration. However, the set-up can operate at a continuous frame rate of $235 \mathrm{~Hz}(117 \mathrm{~Hz})$ in 12- (32-) bit counter mode when using a $1 \mathrm{~Gb} \mathrm{~s}^{-1}$ Ethernet connector to transfer the data from the board to the PC. A frame rate ten times higher can be achieved by continuously streaming data out using a $10 \mathrm{~Gb} \mathrm{~s}^{-1}$ Ethernet connector, while a $6 \mathrm{kHz}$ frame rate is achieved in 12-bit mode by buffering images (8000 images) on the board memories and transferring them out afterwards. These readout modes are commonly used in EIGER for photon applications.

\footnotetext{
${ }^{1}$ The frame rate capability of the chip is maintained in 4- and 8-bit mode, but is presently limited to $6 \mathrm{kHz}$ in 12-bit mode by the readout board communication bandwidth.
}

\subsection{EIGER set-up for LEEM/PEEM}

Fig. 1(a) shows a single $2 \mathrm{~cm} \times 2 \mathrm{~cm}$ readout chip with a bonded sensor glued onto a gold-coated, vacuum-compatible $100 \mathrm{~mm}$-diameter printed circuit board (PCB). The signals in and out of the chip and the high voltage to the sensor are connected to the PCB through wirebonds. The PCB itself acts as a vacuum barrier and is inserted into a DN100CF flange. No active cooling on the chip or sensor is applied. The static power consumption is estimated at $0.83 \mathrm{~W}$ per chip. The standard MCP detector is removed and the EIGER is mounted on the same flange as shown in Fig. 1(b). The EIGER detecting surface is in the same axial position as the MCP detector and no changes of lens settings or magnifications were required. It was necessary to pump the vacuum chamber for 3-4 days to achieve vacuum conditions of 5-8 $\times 10^{-9} \mathrm{mbar}$ and for only one night to $2 \times 10^{-8}$ mbar. As visible from Fig. 1(b), the signals are passed between the PCB and the readout board, which sits outside the vacuum, through flat cables. The readout board is that of a standard EIGER halfmodule, with no modifications. The FPGAs on the board are either air-cooled or water-cooled along the mechanical frame. The power dissipated by the readout board is approximately $45 \mathrm{~W}$. No other custom modifications to the ELMITEC PEEM microscope were made.

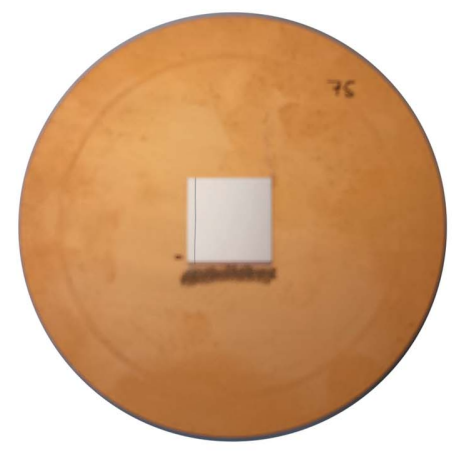

(a)

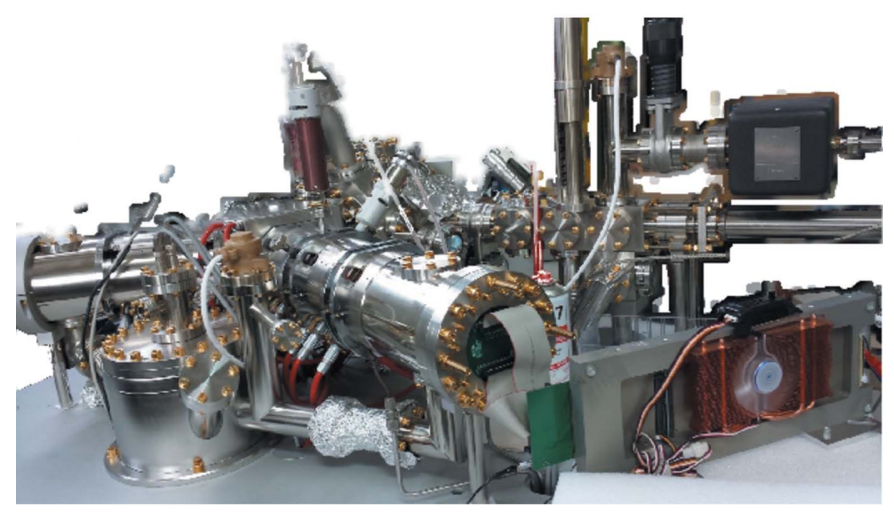

Figure 1

(b)

(a) Photograph of the PCB supporting the detector with a single $(2 \mathrm{~cm} \times$ $2 \mathrm{~cm}$ ) chip. (b) The ELMITEC LEEM III microscope of the SMART II experimental station of the Fritz-Haber-Institute in Berlin with the EIGER installed: the gold-coated side of the PCB is in vacuum and faces the electrons coming from the sample. The readout system is on the bottom right-hand side. The grey flatband cables connect the back of the PCB where the detector is mounted to the readout electronics boards. 


\section{Detector characterization and calibration}

\subsection{Detector calibration with $\mathrm{X}$-rays}

The detector was first characterized in air using monochromatic X-rays. In photon detection, the full photon energy is converted inside the sensor, giving access to an absolute energy calibration. By contrast, electrons lose a significant part of their kinetic energy at the sensor entrance window. The threshold of the readout chip was calibrated versus the energy of monochromatic photons, produced by letting photons from a laboratory X-ray tube impinge on a fluorescence target. The energy resolution of the detector for the considered energies is limited by the noise, which is approximately $400-700 \mathrm{eV}$ root mean square (RMS). In the calibration procedure, we have neglected the $K_{\beta}$ contribution, which is typically below $20 \%$ of the $K_{\alpha}$ contribution. The difference between $K_{\alpha}$ and $K_{\beta}$ is, however, still below the noise-limited energy resolution up to $20 \mathrm{keV}$. The number of counts recorded as a function of the threshold setting can be analytically modelled (Kraft et al., 2009; Tinti et al., 2015) and allows one to calibrate the threshold voltage in terms of the incident beam energy. Fig. 2(a) shows the result of a threshold scan (calibrated to energy) for different photon energies and its derivative is shown in Fig. 2(b). Since the threshold scan is the integral of all the energies above the threshold, its derivative corresponds to the energy distribution. Multiple gain settings have been

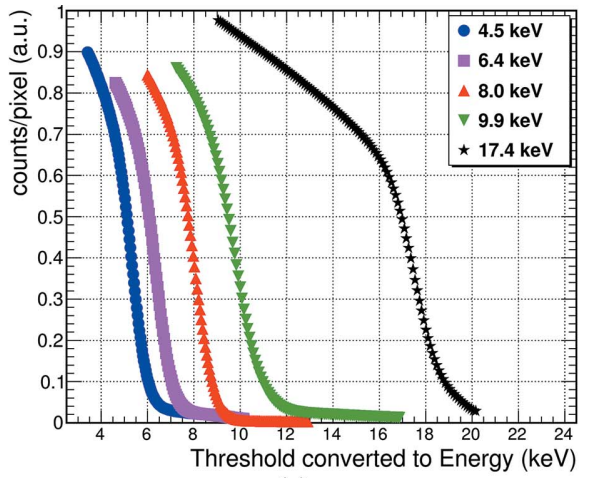

(a)

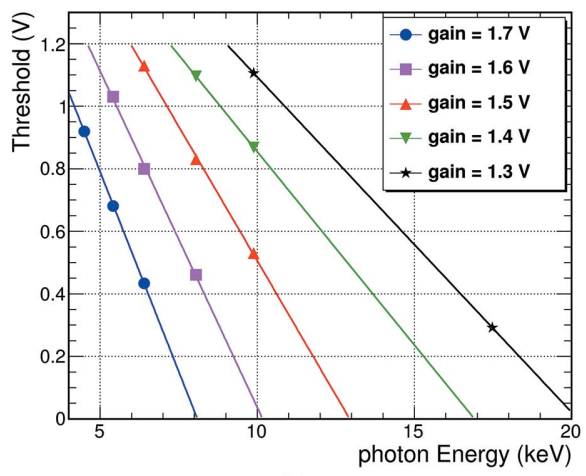

(c)

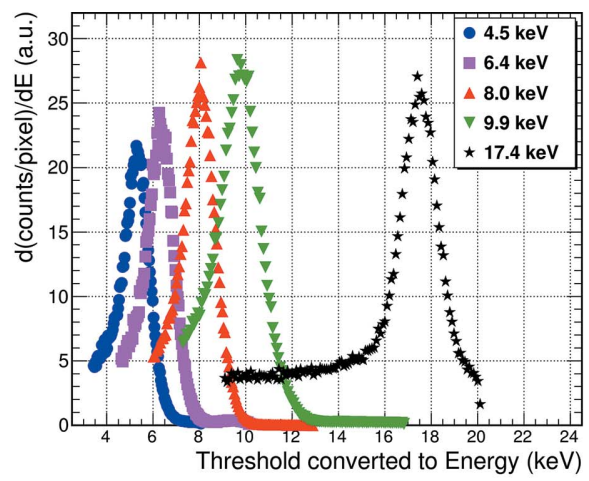

(b)

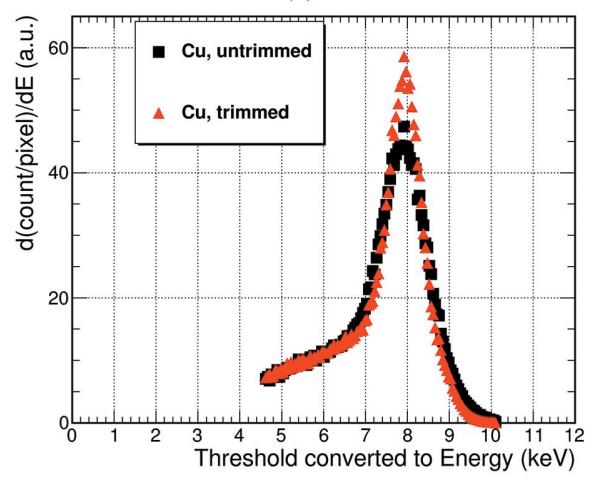

(d)
Figure 2

(a) Threshold scans for monochromatic photons (average of all the pixels), recorded at different photon energies. (b) Derivative of the threshold scans for the average of all pixels. The energy of the peaks corresponds to the $K_{\alpha}$ energy of the fluorescence target used. From left to right: $\mathrm{Cr}, \mathrm{Fe}, \mathrm{Cu}$, Ge and Mo. (c) Threshold-to-energy calibration for the different gain settings. (d) Derivative of a threshold scan for $\mathrm{Cu}$ photons, with 'trimmed' and 'untrimmed' settings (average for all pixels). used to tune the detector performance in the various energy ranges. The obtained threshold-to-energy conversion is plotted in Fig. 2(c). For all the preamplifier gain settings considered, the threshold was confined to within a range where it shows a linear dependence with the photon energy. The charge cloud which is generated in the sensor from the photon interaction diffuses during the drift to the pixel implants. As a consequence, the charge generated from one photon may be collected by more than one pixel, an effect which is referred to as charge sharing. To optimize the spatial resolution and avoid multiple pixels counting the same photon when using a single-photon counter with a monochromatic beam, the threshold should be set such that only pixels with a signal above $50 \%$ of the incoming photon energy count.

The threshold scans are performed using a threshold value which is defined for the entire chip, but small differences in the effective threshold exist among the pixels due to transistor mismatches. In EIGER, there is the possibility to minimize (or 'trim') the pixel-to-pixel threshold differences by introducing a per-pixel 6-bit tuned shift to the chip threshold. The determination of this pixel specific threshold shift was performed using fluorescence photons from a $\mathrm{Cu}$ target. The threshold dispersion is reduced from $320 \mathrm{eV}$ to $55 \mathrm{eV}$ RMS at the energy of $\mathrm{Cu} K_{\alpha}$ photon energy (approximately $8 \mathrm{keV}$ ). However, the energy resolution (obtained as the standard deviation of a Gaussian fit to the peak of the average threshold scan derivative) is only improved from $0.6 \mathrm{keV}$ RMS (for the 'untrimmed' case) to $0.4 \mathrm{keV}$ RMS (for the 'trimmed' one), as shown in Fig. 2(d). Indeed, we expect the energy resolution to be limited by the electronic noise (approximately $0.4 \mathrm{keV}$ RMS at this energy).

\subsection{Detector characterization with electrons}

Electrons in the energy range 8$20 \mathrm{keV}$ interact differently to photons of similar energies in the $\mathrm{Si}$ sensor. Photons have an absorption length of many micrometres and are absorbed throughout the sensor, while electrons have much smaller penetration depths, in the range $1-5 \mu \mathrm{m}$ for $\mathrm{Al}$ and $\mathrm{Si}$ in the 10-20 keV energy range (NIST, 2017). Indeed, electrons, as charged particles, continuously lose energy as they scatter with electrons of the atoms of the material. In addition to the inelastic collisions with the atomic electrons, electrons also undergo multiple elastic Coulomb scattering from nuclei. Each scattering event results in a small deviation to the incoming electron trajectory. As a result, multiple scattering causes a more spatially diffused 
energy deposition along the electron path as electrons lose a variable amount of energy in multiple locations, increasing the possibility to trigger more than one pixel. Since the entrance window of the sensor is composed of an $\mathrm{Al}$ layer and a $\mathrm{Si}$ backplane, electrons lose energy and are scattered by those materials. In addition, since they penetrate only a few micrometres into the depleted sensor, they ionize the $\mathrm{Si}$ and generate a charge cloud close to the sensor backplane. This means that the charge cloud has a long drift range and more opportunity to diffuse. The amount of charge sharing is therefore larger and consequently the events with multiplecounting pixels is increased at low threshold. Since the noise is approximately $400-700 \mathrm{eV}$ RMS for the pre-amplifier gain settings used here, this means that we can reliably set the threshold at five times the noise level, i.e. above $3 \mathrm{keV}$. The electrons with energies considered here $(8-20 \mathrm{keV})$ will stop in the sensor and deposit energies above $3 \mathrm{keV}$ such that EIGER acts as a noise-free detector, since spurious signals arising from the electronic noise are rejected.

Threshold scans have also been performed using electrons originating from an illuminated sample in the PEEM microscope. The electrons are accelerated towards the detector with a known potential. The optics of the microscope have been completely defocused in order to give a homogeneous illumination on the detector surface. The electron energies considered are $8 \mathrm{keV}, 15 \mathrm{keV}$ and $20 \mathrm{keV}$. For a direct comparison, we use the same threshold-to-energy calibration used for photons [Fig. 2(c)]. Fig. 3(a) shows the number of counts as a function of the calibrated threshold setting for different electron energies (full symbols). It is clearly visible that the threshold energy is in all cases at least $3 \mathrm{keV}$ lower than the expected value. The shape of the threshold scan curve also appears very different from the one collected in photon interactions. On the same sensor, on a small area at first and on almost the complete surface at a later time, the $\mathrm{Al}$ layer covering the sensor surface has been chemically removed. ${ }^{2}$ Threshold scans with electrons were repeated after removal of the Al layer, shown in Fig. 3(a) (empty symbols). The shape of the curve changes towards the expected one for photons: the energy threshold is now closer to the expected value as the electrons do not lose energy in the Al. Using values from NIST (2017), electrons with $20 \mathrm{keV}$ energy will lose $2.6 \mathrm{keV}$ upon travelling through $1 \mu \mathrm{m} \mathrm{Al}$, while $10 \mathrm{keV}$ electrons will lose more than $5 \mathrm{keV}$, in good agreement with our results. Fig. 3(b) shows the derivative of the threshold scan in energy. It shows more clearly that, even after the improvement of removing the $\mathrm{Al}$ layer, for $20 \mathrm{keV}$ electrons, the peak of the electron energy is $3 \mathrm{keV}$ lower than the full $20 \mathrm{keV}$ energy, at $15 \mathrm{keV}$ it is approximately $4 \mathrm{keV}$ lower and at $8 \mathrm{keV}$ it is at least $5 \mathrm{keV}$ lower than expected. The removal of the $\mathrm{Al}$ layer allows one to detect these low electron energies, although part of the electron energy is still lost in the entrance window (i.e. the Si backplane).

\footnotetext{
${ }^{2}$ Threshold scans using photons have been retaken to confirm the quality of the high voltage bias of the sensor after the $\mathrm{Al}$ removal.
}

One can apply the pixel-to-pixel threshold correction shifts evaluated using photons to reach more uniformity in the detector. The trim settings used were determined from $\mathrm{Cu} K_{\alpha}$ photons. By calculating and plotting the derivative of the

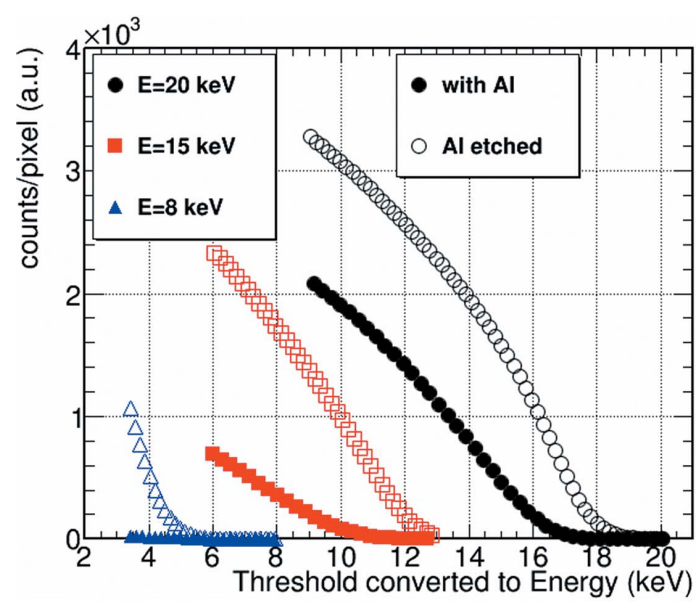

(a)

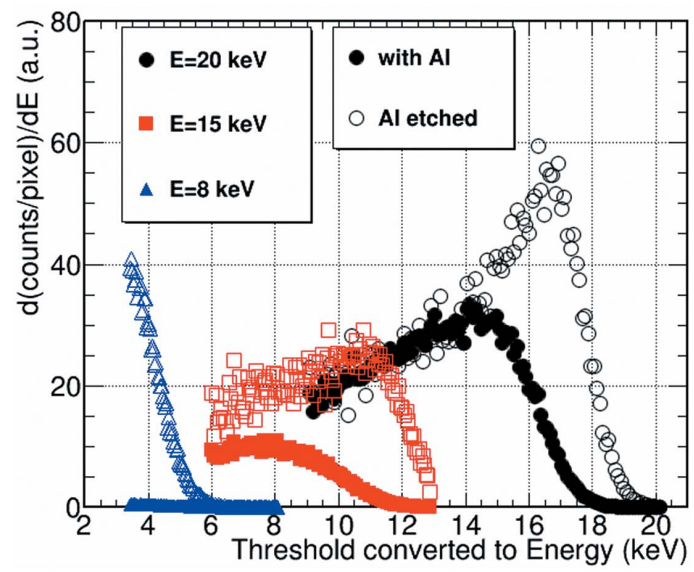

(b)

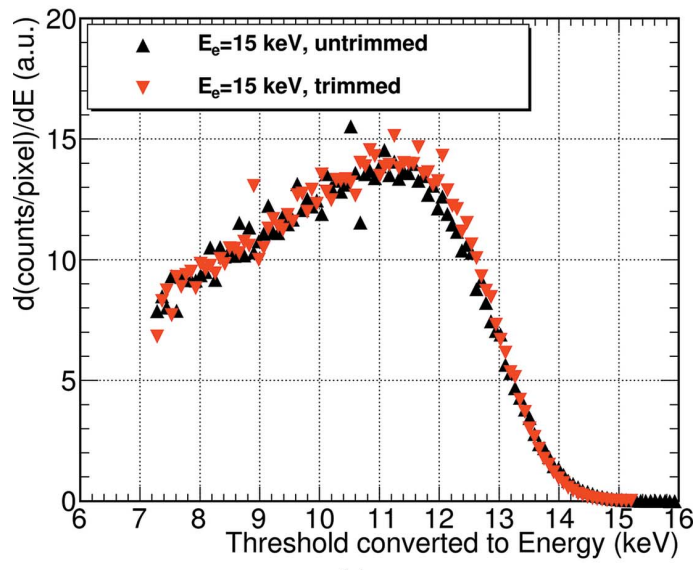

(c)

Figure 3

(a) Threshold scans for electron detection at three different electron energies: $8 \mathrm{keV}, 15 \mathrm{keV}$ and $20 \mathrm{keV}$. Full symbols represent data taken with the standard X-ray Si sensor covered with an Al layer, empty symbols are for the sensor without the Al layer. (b) Derivative of the threshold scan curve. The improvement in the detection of electrons removing the $\mathrm{Al}$ layer is clearly visible. (c) Derivative of a threshold scan for $15 \mathrm{keV}$ electrons in the 'trimmed' and 'untrimmed' settings. 
average threshold scan for $15 \mathrm{keV}$ electrons for the 'trimmed' and 'untrimmed' cases [Fig. 3(c)], one finds that no sizable difference is seen in the energy resolution when reducing the threshold dispersion through the trimming procedure. Therefore the threshold dispersion is not a major contribution to the energy resolution in the case of electron detection (determined instead by noise and a variable energy loss in the entrance window). An estimate of the energy resolution for $15 \mathrm{keV}$ electrons, which deposit a peak energy of approximately $11 \mathrm{keV}$, is obtained by fitting the peak of the threshold scan derivative with a Gaussian function, which gives $1.4 \mathrm{keV}$ standard deviation.

We recorded flat-field images with electrons under similar defocused conditions with $20 \mathrm{keV}$ electrons. We expect, as discussed above, that at this electron energy on average approximately $3 \mathrm{keV}$ are lost at the entrance window of the sensor. To achieve a good spatial resolution, one would set the threshold to $50 \%$ of the energy deposited in the sensor, i.e. approximately at $8.5 \mathrm{keV}$. The flat-field images were recorded at various threshold energies and for both 'trimmed' and 'untrimmed' configurations. For all settings we recorded multiple images which were summed to obtain intensities above $10^{4}$ counts pixel $^{-1}$. The flat-field correction is evaluated for every pixel as the ratio between each pixel intensity and the average number of counts of the detector in order to normalize the individual pixel intensity to the average. The flat-field correction takes care of residual mismatches of threshold inhomogeneities and differences in the pixel sensor efficiency. The residual (non-etched) $\mathrm{Al}$ region at the edge of the sensor is masked in the analysis. We evaluated the size of the corrections as a function of the different threshold settings, and indeed we verified that lower corrections are required when setting the threshold to $8.5 \mathrm{keV}$. In addition, the RMS of the distribution of the correction is reduced from $3.4 \%$ to $2.3 \%$ by applying the pixel-to-pixel trimming settings. The flatfield correction maps at threshold $8.5 \mathrm{keV}$ is shown in Fig. 4(a) for the 'untrimmed' case, while Fig. $4(b)$ is for the 'trimmed' case. The homogeneity is visibly enhanced by applying the trimming correction. On the other hand, some structures in the sensor that are not clearly visible in the 'untrimmed' distribution start to be noticeable. We see the presence on the sensor of some segments of rings, which we attribute to small inhomogeneities in the doping of the sensor. This effect is present in every image we recorded with the trim settings, but this feature can be removed from the image by applying a flatfield correction. On top of the segments of rings, we see also a gradient in the number of counts over the sensor. This effect is due to the remaining presence of the sample imprint despite the defocusing magnification used. Other flat-field images were recorded for different defocusing settings and do not manifest the same gradient; however, they were recorded with smaller statistics (not shown). Comparing the above finding with flat-field normalization images for MCPs, we find that the performance of the EIGER detector provides the following improvements: (i) the RMS of the counts of the MCPs is typically greater than $10 \%$ of the mean as compared with $2.3 \%$ for EIGER; (ii) the flat-field correction for the MCPs varies in time due to the beam exposure, an effect to which EIGER is immune, as discussed in the next paragraph.

In contrast to MCPs, Si sensors do not suffer from radiation damage for low-energy $(<20 \mathrm{keV})$ electron interactions. The interaction region of electrons at such low energies as the ones used in LEEM/PEEM is limited to a few micrometres into the Si. The energy of the electrons considered in LEEM/PEEM applications is too low to cause lattice displacements (i.e. damage in the Si sensor) given that a minimum electron energy of $260 \mathrm{keV}$ is required to remove a silicon atom from its lattice position (Rossi et al., 2006). The other mechanism whereby electrons could induce damage in the sensor is by increasing the leakage current through an increase in the oxide positive charge (Rossi et al., 2006). However, in the EIGER sensor, the back side is not structured and therefore no oxide is present. The readout chip is protected by the sensor and it is not irradiated. For these reasons, we expect

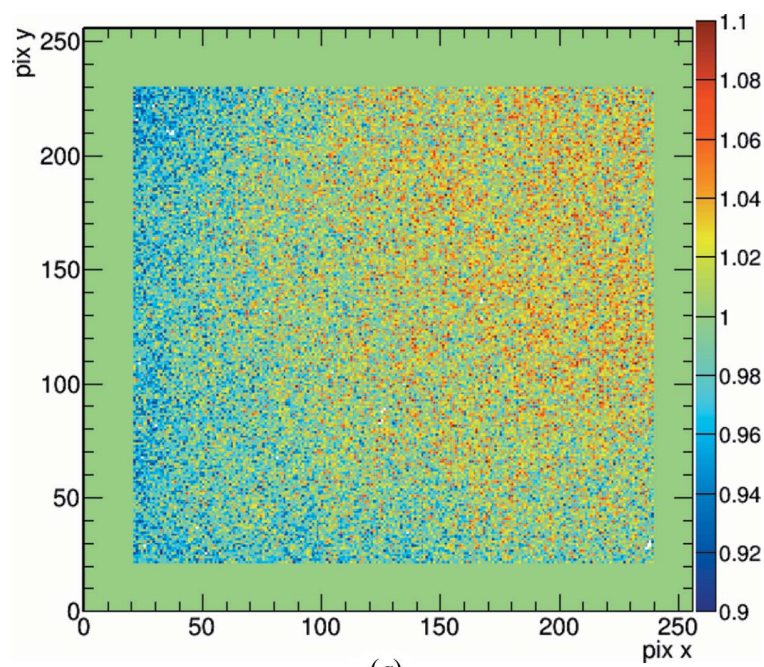

(a)

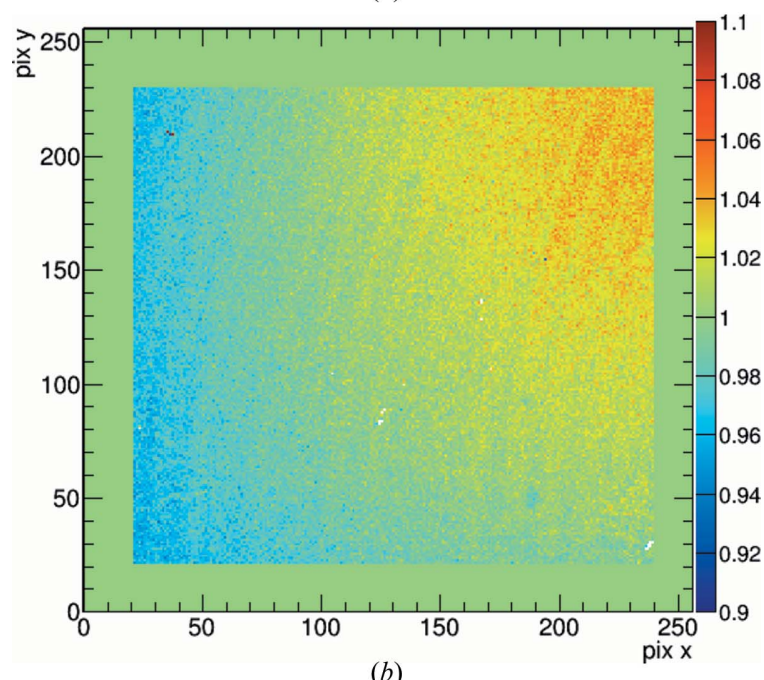

Figure 4

(a) Map of the 'untrimmed' flat-field correction for the EIGER detector (where the area at the edge where the $\mathrm{Al}$ has not been completely etched away has been masked), with threshold set at $8.5 \mathrm{keV}$. (b) Corresponding flat-field correction map for the detector, with threshold set at $8.5 \mathrm{keV}$ and 'trimmed' configuration. 
that EIGER used for LEEM and PEEM experiments will not suffer from degradation due to radiation damage.

\section{EIGER performance measurements with UV-PEEM and LEEM}

The imaging performance with EIGER as a detector for UVPEEM/LEEM was carried out at the SMART II experimental station of the Fritz-Haber-Institute in Berlin, which operates an ELMITEC LEEM III instrument, installed at the Helmholtz-Zentrum Berlin (HZB), shown in Fig. 1(b). In order for the EIGER detector to be a successful replacement candidate for the MCP-based system it must: (i) deliver live images (for instance to align the instrument) and allow image recording, (ii) have a number of resolving elements comparable with or better than the MCPs, (iii) offer a higher dynamic range and improved signal-to-noise ratio, and (iv) be easy to use. The typical frame rates for live imaging with the MCPs are $10 \mathrm{~Hz}$. As already discussed above, the EIGER detector supersedes the MCPs performance by acquiring images at a rate up to $6 \mathrm{kHz}$ using 12-bits and even higher rates with smaller counters. Although the CCD cameras used with the MCPs allow for higher acquisition rates, the higher electron fluxes necessary to provide sufficient image signal would instantly deteriorate the MCP. This is not the case for the EIGER, where higher fluxes with high frame rates can be used effectively without damage to the detection system.

The MCPs have a lateral resolution of $\sim 110 \mu \mathrm{m}$ (Moldovan et al., 2008) and a diameter of $\sim 40 \mathrm{~mm}$, therefore in each image up to $\sim 300$ elements may be resolved along the diagonal. For a pixel image sensor such as EIGER, the smallest resolvable space between two objects (or highest spatial frequency) is the inverse of the Nyquist frequency, which is twice the pixel size, or in our case $150 \mu \mathrm{m}$. Image features at the detector plane smaller than $150 \mu \mathrm{m}$ may still be resolved, but might result in aliasing effects. Fig. 5(a) shows a direct UVPEEM image of a monolayer of graphene on a Ge buffer layer on $\mathrm{SiC}(0001)$ (Emtsev et al., 2011). No trimming or flat-field correction is applied. Whereas the low-frequency intensity variations are due to workfunction differences, the thin dark lines running mainly along the vertical direction are due to terrace edges. The image is obtained by averaging a stack of 50 images of $10 \mathrm{~s}$ each. The image in Fig. 5(a) is undersampled and most thin dark lines are smaller than the size of the single EIGER pixel. This is shown in Fig. 5(b) where two profiles across two thin dark lines are shown to be two or one pixel wide. This confirms that, with EIGER, features at the detector as small as $75 \mu \mathrm{m}$ may be resolved.

The dynamic range of a MCP-based system depends on the gain, defined by the bias voltage, and is limited by noise at low count rates and by the onset of irreversible damages at higher count rates. An accurate estimation of the dynamic range of the MCPs is challenging and here we provide an upper limit for the MCP dynamic range by considering normal operating conditions. Typical LEEM and PEEM set-ups operate at bias voltages between $1.1 \mathrm{keV}$ and $1.3 \mathrm{keV}$. At these bias voltages, the maximum detectable current is limited between $5 \mathrm{nA}$ and
$0.08 \mathrm{nA}$, respectively, and, given an MCP diameter of $40 \mathrm{~mm}$, the maximum flux is between 0.4 and 25 Mcounts $\mathrm{mm}^{-2} \mathrm{~s}^{-1}$. A key feature of the EIGER detector is that its dynamic range is only limited by the counter size of $2^{32}$ (or $4 \times 10^{9}$ counts). There is a flux intensity above which the counting capability of EIGER (as a single-particle-counting detector) loses linearity as the particles arrive at too high a rate to leave enough time for the signal to return to below the threshold. The rate of detected particles $n_{\mathrm{d}}$ follows the paralizable counter model: $n_{\mathrm{d}}=n_{\mathrm{i}} \exp \left(-n_{\mathrm{i}} \tau\right)$, where $n_{\mathrm{i}}$ is the incident rate and $\tau$ is a deadtime, in the range $150-600 \mathrm{~ns}$ according to the preamplifier gain settings. Indeed, the more the signal is amplified, the wider the pulse and the longer it will take to return under threshold. The incident flux at which there is a $10 \%$ loss in linearity has been measured with photons to be 0.2-0.7 Mcounts pixel ${ }^{-1} \mathrm{~s}^{-1}$ (32-126 Mcounts $\left.\mathrm{mm}^{-2} \mathrm{~s}^{-1}\right)$. At higher incident fluxes (up to $\sim 1-4$ Mcounts $_{\text {pixel }}^{-1} \mathrm{~s}^{-1}$,

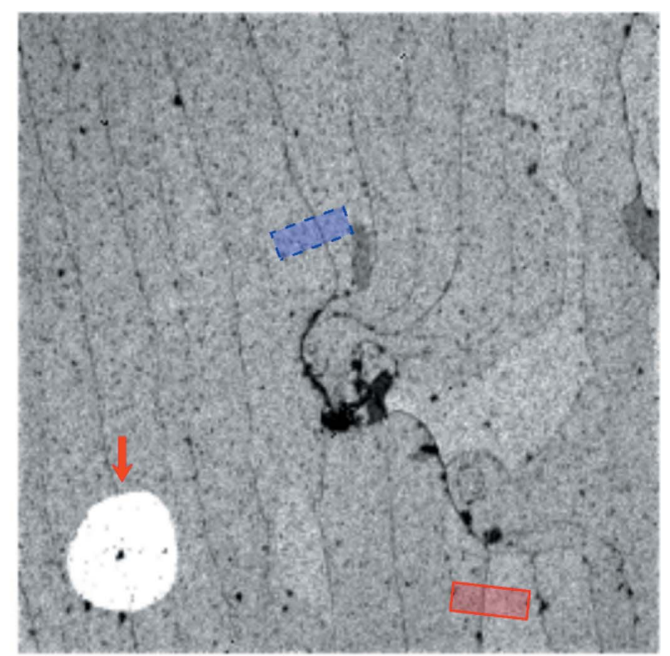

(a)

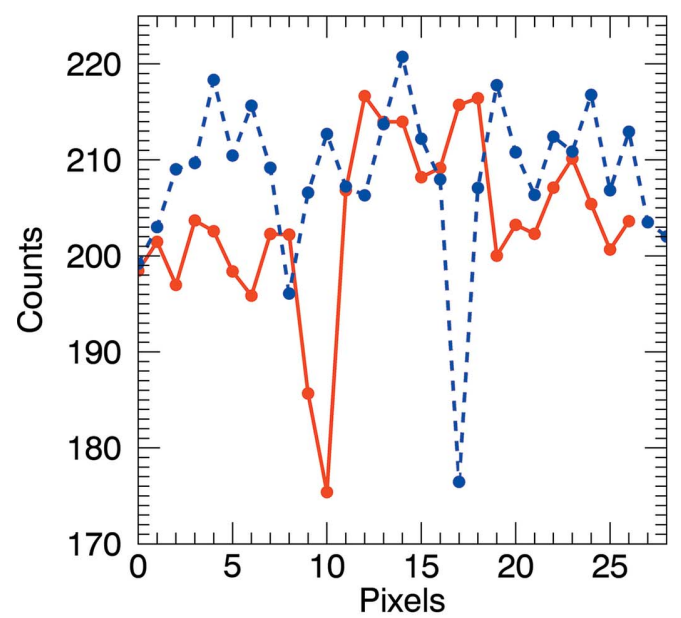

(b)

Figure 5

UV-PEEM image of a monolayer of graphene on a Ge buffer layer on $\mathrm{SiC}(0001)$. The field of view is $25 \mu \mathrm{m} \times 25 \mu \mathrm{m}$. The red arrow in the bottom-left highlights the detector region with a thinned entrancewindow as discussed in \$3.2. (b) Line profiles across the long axis of the two boxes with solid and dashed lines, by averaging along the perpendicular direction (each 11 pixels wide) as shown in $(a)$. 
or $\sim 180-700$ Mcounts $\mathrm{mm}^{-2} \mathrm{~s}^{-1}$ ), the detector counts up to a maximum counter value of, respectively, 0.6-2.4 Mcounts pixel ${ }^{-1} \mathrm{~s}^{-1}$ (108-420 Mcounts $\left.\mathrm{mm}^{-2} \mathrm{~s}^{-1}\right)$, after which the detected counts start to decrease with more flux. Algorithms to correct for the EIGER non-linearity can be applied both online and offline (Sobott et al., 2013; Johnson et al., 2014), up to an incident flux corresponding to the detected maximum. However, there is a variation in pixel-topixel dead-times, while the correction is applied for an average dead-time, hence it is advantageous to limit the incident flux to less than $10 \%$ loss in linearity to limit the spread in pixel differences to the few-percent level. The estimate given above shows that the flux tolerated by EIGER is significantly higher than the flux to which one can expose the MCPs.

The high dynamic range of the EIGER detector is demonstrated in Fig. 6(a), which shows a low-energy electron diffraction (LEED) pattern of a $(7 \times 7)$-Si(111) (Schlier \& Farnsworth, 1959; Binnig et al., 1983) surface acquired in $4 \mathrm{~s}$ in the LEED mode of SMART II. The investigated sample surface was not perfectly clean in the present performance measurements and the LEED pattern exhibits a high background intensity. For this reason the pattern is displayed using logarithmic intensity scaling to make the $(7 \times 7)$ reconstruction diffraction spots more visible. The line profile taken along the red elongated box shown in Fig. 6(a) is shown in Fig. 6(b). The highest intensity reached at the $(00)$ reflection spot is $2.5 \times 10^{5}$ counts and the lowest, outside the Ewald sphere, has 0 counts. The non-zero counts outside the Ewald sphere from pixels 280 to 286 , shown in the inset, are probably due to slight instrumental misalignment or scattering of electrons within the microscope and are unlikely to be due to detector noise. The high dynamic range of the EIGER is advantageous specifically for quantitative analysis of electron diffraction spot profiles where the signals span many orders of magnitude and require excellent signal-to-noise ratios. We found in further experiments, not shown here, that in $50 \mathrm{~min}$ of acquisition with the microscope turned off the only signal detected was consistent with cosmic rays. The corresponding count histogram reveals that less than $3 \%$ of the pixels have more than one count and only about ten out of 65536 pixels have detected two counts.

\section{EIGER performance measurements with X-PEEM}

In X-ray photoemission electron microscopy (X-PEEM) the samples are illuminated with intense, tunable synchrotron radiation (Bauer, 2014). The X-PEEM imaging mode allows one to perform spatially resolved X-ray absorption spectroscopy to obtain local chemical information. Magnetic or ferroelectric phenomena with sensitivity down to the single monolayer regime and below can be probed employing resonantly enhanced linear or circular dichroic effects occurring when exciting certain absorption edges with polarized X-rays. The spatial resolution of X-PEEM in imaging mode is limited to about $50 \mathrm{~nm}$ due to chromatic aberrations in conventional instruments. It can be further reduced to almost $15 \mathrm{~nm}$ in

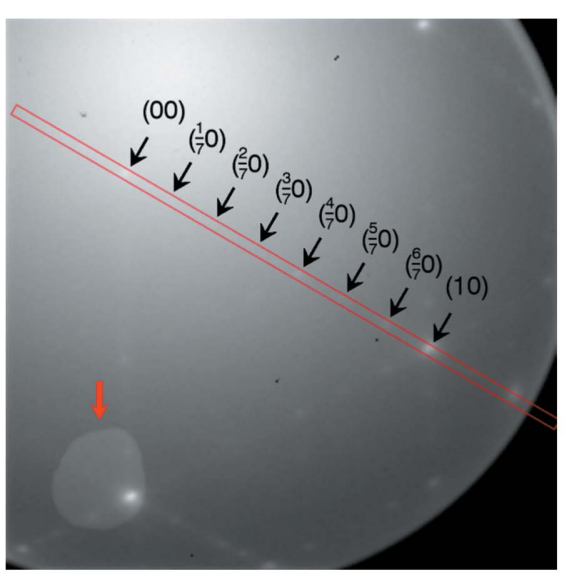

(a)

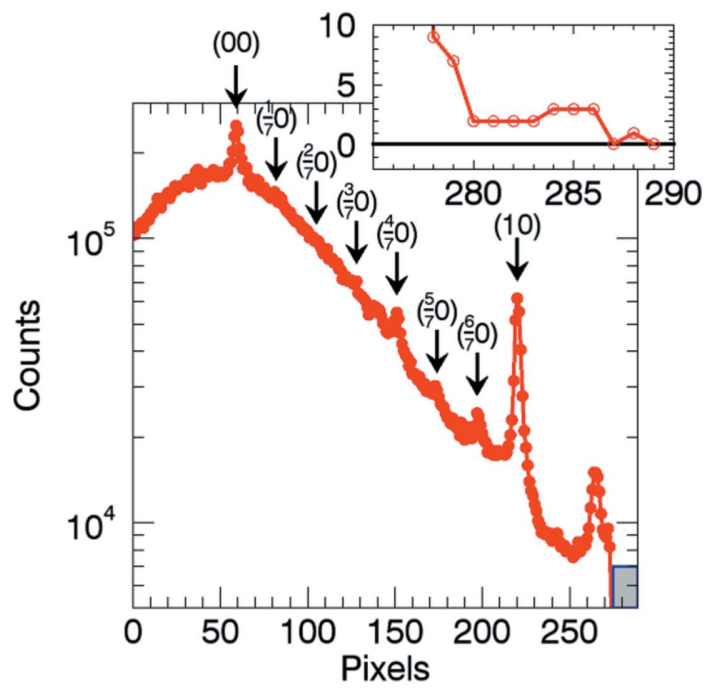

(b)

Figure 6

(a) Image of the LEED pattern of a $(7 \times 7)$ reconstructed $\mathrm{Si}(111)$ surface. The kinetic energy of the electrons was set to $29.4 \mathrm{eV}$. The LEED-image intensity has been logarithmically rescaled. The two outer arrows, along the elongated red box, indicate the (00) elastic reflected beam and the (10) first-order diffraction spot. The other arrows in between are distributed at integer multiples of a seventh of the (00)-(10) distance. The red arrow in the bottom-left highlights the detector region with a thinned entrance-window as discussed in \$3.2. (b) Logarithmic plot of the line profile across the long axis of the elongated red box as shown in $(a)$. The black arrows mark the same positions as in $(a)$. The bottom-right of the plot, for counts below 10, is expanded in the linear plot in the inset. Notice that the line profile outside of the Ewald's sphere eventually drops to zero counts.

aberration-corrected instruments, which also have an improved transmission (Schmidt et al., 2013).

Most X-PEEM instruments are equipped with an additional energy analyzer, which can be used, for instance, to perform local microspot X-ray photoelectron spectroscopy. For imaging, the analyzer is usually set so that the secondary photoelectrons can pass and impinge on the detector. Given the high photon flux of undulator beamlines, high signal intensities can be easily achieved in the imaging but also in the spectroscopic modes. Moreover, the signal intensity in $\mathrm{X}$-PEEM depends on the X-ray absorption cross section 
which, in addition to the element-specific resonances, depends on the atomic number $Z$. Thus, the X-PEEM signal can become very high for heavy elements such as Au or Pt even without resonant excitation. As a consequence, X-PEEM imaging has frequently to deal with sample features of very different intensities. Thus, similar to LEEM and UV-PEEM, the different operation modes and the strong intensity variations in typical samples call for a detector with high dynamic range, high signal-to-noise ratio, and which is robust against exposure to high electron intensities.

The EIGER performance has been tested in an X-PEEM experiment at the SIM beamline at the SLS (Flechsig et al., 2010; Guyader et al., 2012). As a test sample we chose Fe nanoparticles with sizes between 8 and $20 \mathrm{~nm}$ deposited onto $\mathrm{Si}$ wafers with $\mathrm{Au}$ marker structures (Kleibert et al., 2007). The latter are used as references for alignment of the microscope and for identifying the very same nanoparticles in different instruments for complementary microscopy investigations. In these studies, X-PEEM is used for acquiring X-ray absorption spectra of individual nanoparticles and for magnetic characterization by means of the X-ray magnetic circular dichroism (XMCD) effect (Rodríguez et al., 2010; Vaz et al., 2014). Such samples are challenging for X-PEEM, since they are characterized by a low signal amplitude for individual nanoparticles on top of a high signal background from the $\mathrm{Si}$ substrate and a larger signal from the Au marker structures. This combination requires finding a compromise for the signal intensity for MCP detection and these samples are therefore ideally suited for testing an alternative detector concept. Besides carrying out the EIGER performance tests, our interest was to investigate the magnetic properties of the $\mathrm{Fe}$ nanoparticles upon exposure to ambient air following a controlled oxidation process performed earlier on the same sample (Vaz et al., 2014).

Fig. 7 shows X-PEEM images of the nanoparticle sample recorded with the photon energy set to the iron $L_{3}$-edge, at about $709 \mathrm{eV}$. The left-hand column shows the MCP detector images, while the right-hand column shows the EIGER images. To facilitate a direct comparison of both detectors we have chosen the same data acquisition time, which results in about 2000 counts on the bare $\mathrm{Si}$ substrate surface. The EIGER was used with a 12-bit counter to compare with the MCP data, which are acquired with a 12-bit CCD camera. For EIGER, trimming has been applied with a $8.5 \mathrm{keV}$ threshold. With these settings the lithographically made gold marker structures appear in both images in Figs. 7(a) and 7(b) as the brightest features, which are moreover saturated due to their high X-ray absorption cross section when compared with the $\mathrm{Si}$ substrate or the nanoparticles. Although the latter are resonantly excited they appear only as less bright spots in both detectors, visible only after adjusting the contrast level, Figs. $7(c)$ and $7(d)$. However, this signal level (typically $5-10 \%$ above the substrate background, as illustrated in the line scans shown in the insets to Fig. 7) is sufficient to acquire X-ray absorption spectra and to achieve good magnetic contrast by means of the XMCD effect. Each spot corresponds typically to the signal of an individual nanoparticle. When compared with the EIGER detector, Fig. 7(d), the MCP image in Fig. 7(c) shows much less contrast. This is due to spatial inhomogeneities in the MCP detector, which give rise to local variations in intensity that are similar in magnitude to the nanoparticle signal. This is clearly demonstrated in the line scans in the respective figure insets, where the MCP background signal arising from the detector inhomogeneities are of comparable amplitude with the signal from the nanoparticles. As a quantitative electron-counting device the EIGER does not suffer from inhomogeneities and thus delivers a good contrast and a
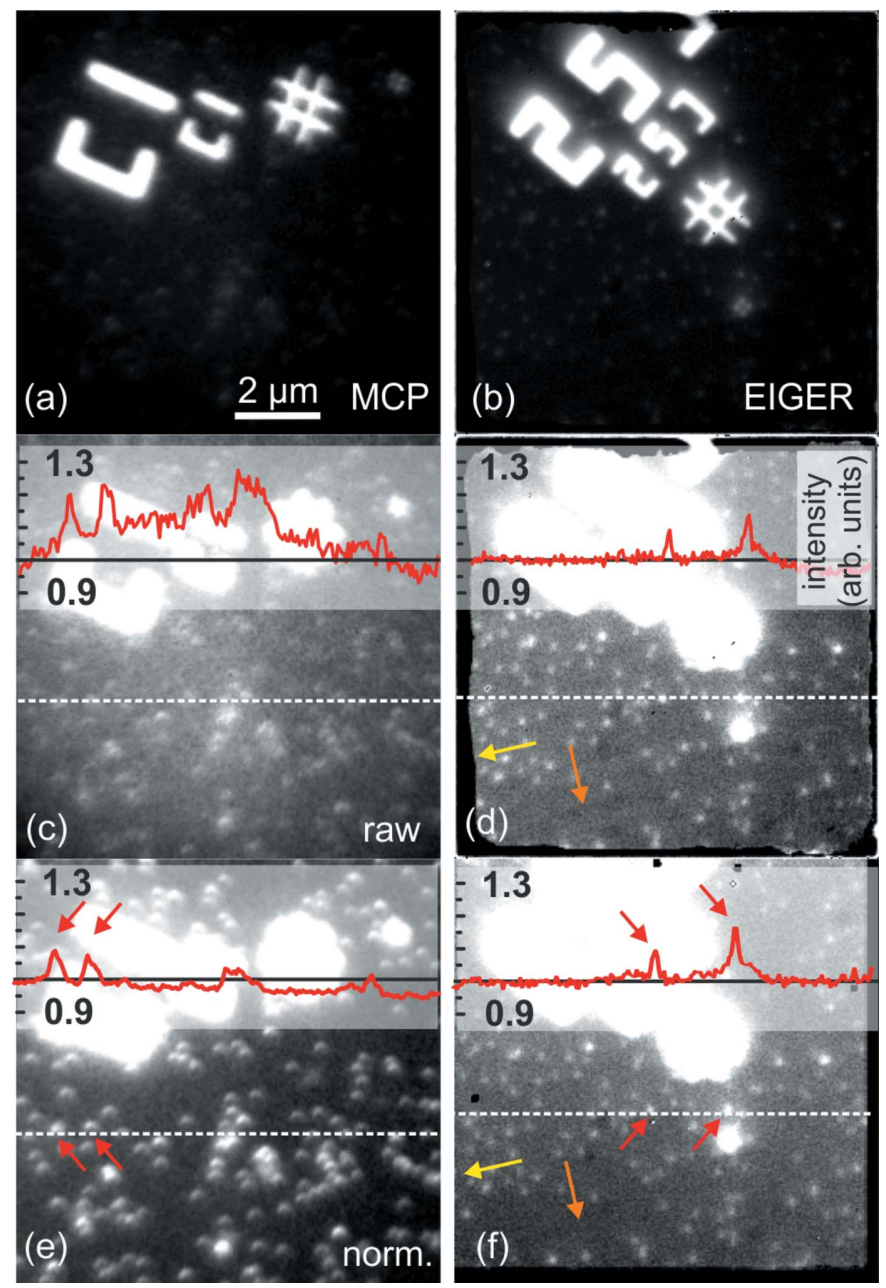

Figure 7

X-PEEM images of supported iron nanoparticles recorded with the X-ray photon energy set to the Fe $L_{3}$-edge at about $709 \mathrm{eV}$ using the MCP (left column) and the EIGER (right column). (a)-(b) Images with contrast optimized to observe the Au marker structures. Nanoparticles are seen in both images as fainter spots. $(c)-(d)$ The same images but with the contrast optimized to visualize the nanoparticle signal. Insets show line scans extracted along the dashed lines and normalized to the substrate intensity. $(e)-(f)$ Flat-field and drift-corrected images with line scans in the insets. For the MCP detector this processing leads to a much better contrast of the nanoparticles, while it gives only a marginal improvement for the EIGER. The red arrows indicate the signal of two nanoparticles. Yellow arrows highlight some artefacts in the EIGER detector image originating from the residual $\mathrm{Al}$ layer and their removal by the flat-field normalization. Orange arrows highlight the removal of subtle inhomogeneities of the EIGER detector. The dark areas at the bottom and the right-hand side of $(f)$ are not EIGER detector features but result from the sample drift correction. 
much better visualization of the nanoparticles directly in the raw X-PEEM images, as seen in Fig. 7(d).

In order to deal with the MCP detector inhomogeneities, one typically acquires a flat-field image with a strongly defocused objective lens setting to achieve a featureless bright image. The latter is then used for normalization by means of pixel-wise division of a raw X-PEEM image [as shown in Fig. 7(c)] with the flat-field image. The result of such a normalization is shown in Figs. 7(e) and 7(f) for both detectors. For the MCP image this results in a significant improvement of contrast, while for the EIGER detector such a procedure has only a minor effect, because of its intrinsic background-free counting mode. This is again evident in the line scans shown in Fig. 7, where the normalization procedure for the MCP removes most of the background contribution to reveal the nanoparticle signal, while for the EIGER such a procedure is less critical and the most visible effect is that of cancelling the signal attenuation introduced by the remaining Al layer at the edges of the detector, as indicated by the yellow arrows in Figs. 7(d) and 7(f), and the removal of faint stripe features from slight doping inhomogeneities in the detector, as shown by the orange arrows [cf. Fig. 4(b)]. Such a low background signal in the EIGER is particularly useful for real-time imaging, where the small signal from features in the sample can be directly visualized. Another improvement of the data quality is achieved by recording and processing another set of data with the photon energy set at a few $\mathrm{eV}$ below the resonant absorption edge, e.g. to $703 \mathrm{eV}$ in the present case. Pixelwise division of normalized and drift-corrected images recorded at both energies, with the photon energy set to the iron $L_{3}$-edge and below, yields so-called X-ray PEEM elemental contrast maps. Fig. 8 shows such maps recorded $(a)$ with the standard MCP detector used in an ELMITEC microscope and (b) with EIGER. Since this operation removes all contrast which does not originate from $\mathrm{Fe}$, the nanoparticles are now clearly visible in both detectors. In both images one can clearly observe artefacts which result from the saturated signals of the Au marker structures. While with MCP this is unavoidable with this type of sample, the EIGER detector can be used to circumvent this issue, by choosing a 32bit counter instead of the 12-bit counter, which provides an increase in dynamic range by a factor $10^{6}$.

Finally, we demonstrate the performance of both detectors when recording magnetic contrast images. For this purpose, the photon energy is set to the iron $L_{3}$-edge and two images are acquired with circularly left- and right-handed polarized light. The XMCD effect leads then to a helicity- and magnetization-dependent variation in the absorption intensity of the individual nanoparticles (Stöhr, 1995; Balan et al., 2014). Magnetic contrast maps are obtained from pixelwise division of normalized and drift-corrected X-PEEM images acquired with opposite helicities. Similar to the elemental contrast images, this procedure leads to a cancellation of all nonmagnetic contributions on the sample. As a result one finds in both images in Figs. $8(c)$ and $8(d)$ magnetic contrast ranging from white to black depending on the magnetization of the individual nanoparticle. We observe that the EIGER data
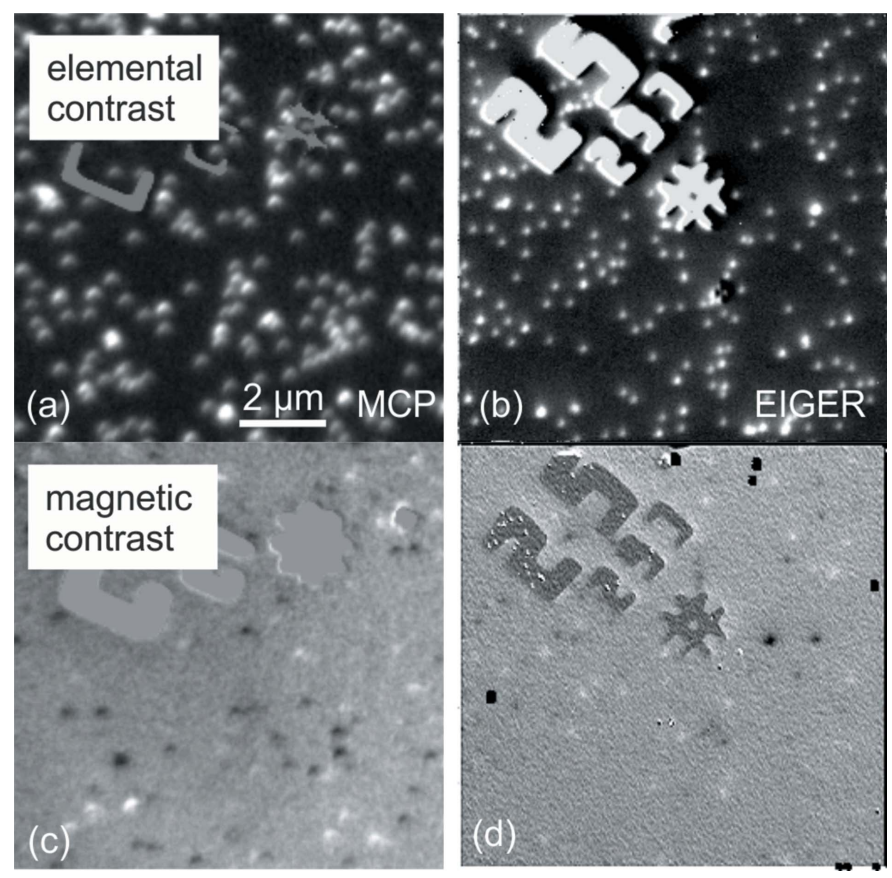

Figure 8

Drift-corrected X-PEEM images of supported iron nanoparticles recorded using the MCP (left column) and the EIGER (right column). (a) -(b) Elemental contrast maps, in which the signal of the Fe-containing parts of the sample is enhanced with both detectors. $(c)-(d)$ Magnetic contrast maps obtained from flat-field and drift-corrected X-PEEM images recorded with right- and left-circularly polarized light.

reveal a remarkably large number of particles showing magnetic contrast, indicating that the exposure to ambient air did not affect the unusual high anisotropy state found previously in these particles (Balan et al., 2014). The detection of magnetic contrast further confirms the presence of a metallic core (Pratt et al., 2014; Vaz et al., 2014), which is assigned to a passivation of the nanoparticles achieved by the controlled oxidation performed in the previous experiments carried out with the MCP detector (Vaz et al., 2014; Lu et al., 2007).

In summary, the EIGER yields data quality which is similar or superior to the conventional MCP detectors used for $\mathrm{X}$-PEEM. Its particular strength is the high dynamic range, which enables the investigation of samples which possess features with large intensity differences. Its homogeneous background is a huge advantage when attempting to focus on objects with weak intensity, such as the present nanoparticles, in real-time. Moreover, the background is constant in time which is often not the case for the MCP. Finally, the EIGER avoids the danger of detector damage by overexposure, which makes the instrument robust for general user experiments carried out at large-scale synchrotron facilities.

\section{Conclusions and prospects for a dedicated EIGER PEEM set-up}

In this paper we report the use of the EIGER Si hybrid pixel detector as a detector for low-energy electrons in PEEM 
experiments. EIGER is characterized by a small pixel size $(75 \mu \mathrm{m} \times 75 \mu \mathrm{m})$ and high frame rate (up to $23 \mathrm{kHz}$ ). While EIGER has been optimized for photon detection, it is very suitable for electron detection with only minimal modifications to the sensitive part of the detector. In particular, we show that removal of the $\mathrm{Al}$ protection layer is the key to achieving good sensitivity for EIGER. The detector settings have been studied and optimized for electrons: it has been shown that the optimal detector threshold setting is at half the electron energy minus the energy lost in the backplane. It is advantageous to apply pixel-to-pixel corrections to homogenize the threshold on the whole detector. In addition, one can further improve the image by applying a flat-field correction. The major advantage compared with a standard MCP detector is the higher signal-to-noise ratio, which allows direct imaging even of small structures without the need of applying real-time corrections, resulting in an easier sample alignment procedure and faster judgment of the image quality in real time. In addition, the detector has a dynamic range of 32-bits thanks to the on-board image summation. The very high frame rate $(23 / 12 / 6 \mathrm{kHz}$ in $4 / 8 / 12$ bit mode) provides time resolution for real-time imaging and short exposures for radiation-sensitive samples.

We have further demonstrated that EIGER can be employed successfully as a detector for PEEM and we envisage that the following additional developments will make EIGER very attractive for electron and X-ray spectromicroscopes:

(i) The sensitive area can be enlarged ${ }^{3}$ from $2 \mathrm{~cm} \times 2 \mathrm{~cm}$ to $4 \mathrm{~cm} \times 4 \mathrm{~cm}$, or $256 \times 256$ pixels to $512 \times 512$ pixels. This improvement is possible by tiling together $2 \times 2$ readout chips (quad) and bonding them to a single $4 \mathrm{~cm} \times 4 \mathrm{~cm}$ sensor. There is no dead area within the quad geometry thanks to the double/quadruple-size sensor pixels at the boundaries of the readout chips. Interpolation of the counts for these edge pixels with the neighbouring pixels is possible. The standard EIGER readout set-up is already suitable for readout up to four chips, so only a simple software adaptation for the display of the quad geometry is needed.

(ii) A new sensor has been designed for the quad and has been optimized to have a thin backplane without the $\mathrm{Al}$ layer to provide an overall thin entrance window of approximately $200 \mathrm{~nm}$, which is suitable for low-energy $(8-20 \mathrm{keV})$ electrons and low-energy photons. A backplane thickness of $200 \mathrm{~nm}$ would allow electrons of $20 \mathrm{keV}(10 \mathrm{keV})$ to lose only $0.5 \mathrm{keV}$ $(0.9 \mathrm{keV})$ (NIST, 2017) before being detected. The sensor thickness will be maintained at $320 \mu \mathrm{m}$, although a thinner sensor is a possibility for future upgrades for higher spatial resolution by reducing the diffusion of charge cloud (and charge sharing) during drifting.

As the dimensions of the EIGER quad adapt well to the field of view normally used by the ELMITEC electron microscopes, permanent EIGER set-ups are foreseen.

\footnotetext{
${ }^{3}$ A first set-up is being produced at the time of writing.
}

\section{Acknowledgements}

Part of this work was performed at the Surface/Interface Microscopy (SIM) beamline of the Swiss Light Source, Paul Scherrer Institut, Villigen, Switzerland, and part at the SMART II experimental station of the Fritz-Haber-Institute in Berlin, which is funded by General Administration of the Max-Planck-Society. HM would like to thank Alexei Zakharov for providing the sample measured in Fig. 5.

\section{References}

Amsterdam Scientific Instruments (2017). Electron microscopy, http://www.amscins.com/expertise/electron-microscopy.

Anders, S., Padmore, H. A., Duarte, R. M., Renner, T., Stammler, T., Scholl, A., Scheinfein, M. R., Stöhr, J., Séve, L. \& Sinkovic, B. (1999). Rev. Sci. Instrum. 70, 3973-3981.

Balan, A., Derlet, P. M., Rodríguez, A. F., Bansmann, J., Yanes, R., Nowak, U., Kleibert, A. \& Nolting, F. (2014). Phys. Rev. Lett. 112, 107201.

Bauer, E. (2014). Other Surface Imaging Methods with Electrons. New York: Springer.

Binnig, G., Rohrer, H., Gerber, C. \& Weibel, E. (1983). Phys. Rev. Lett. 50, 120-123.

Casanas, A., Warshamanage, R., Finke, A. D., Panepucci, E., Olieric, V., Nöll, A., Tampé, R., Brandstetter, S., Förster, A., Mueller, M., Schulze-Briese, C., Bunk, O. \& Wang, M. (2016). Acta Cryst. D72, 1036-1048.

Cheng, X. M. \& Keavney, D. J. (2012). Rep. Prog. Phys. 75, 026501.

Dectris (2017). Now detecting electrons too!, https://www.dectris.com/ news.html\#now-detecting-electrons-too.

Dinapoli, R., Bergamaschi, A., Henrich, B., Horisberger, R., Johnson, I., Mozzanica, A., Schmid, E., Schmitt, B., Schreiber, A., Shi, X. \& Theidel, G. (2011). Nucl. Instrum. Methods Phys. Res. A, 650, 7983.

Emtsev, K. V., Zakharov, A. A., Coletti, C., Forti, S. \& Starke, U. (2011). Phys. Rev. B, 84, 125423.

Engel, W., Kordesch, M., Rotermund, H., Kubala, S. \& von Oertzen, A. (1991). Ultramicroscopy, 36, 148-153.

Flechsig, U., Nolting, F., Fraile Rodriguez, A., Krempasky, J., Quitmann, C., Schmidt, T., Spielmann, S. \& Zimoch, D. (2010). AIP Conf. Proc. 1234, 319.

Fraile Rodríguez, A., Kleibert, A., Bansmann, J., Voitkans, A., Heyderman, L. J. \& Nolting, F. (2010). Phys. Rev. Lett. 104, 1 27201.

Gastel, R. van, Sikharulidze, I., Schramm, S., Abrahams, J. P., Poelsema, B., Tromp, R. M. \& van der Molen, S. J. (2009). Ultramicroscopy, 110, 33-35.

Gimenez, E. N., Ballabriga, R., Blaj, G., Campbell, M., Dolbnya, I., Frodjh, E., Horswell, I., Llopart, X., Marchal, J., McGrath, J., Omar, D., Plackett, R., Sawhney, K. \& Tartoni, N. (2015). IEEE Trans. Nucl. Sci. 62, 1413-1421.

Griffith, O. H. \& Engel, W. (1991). Ultramicroscopy, 36, 1-28.

Guizar-Sicairos, M., Johnson, I., Diaz, A., Holler, M., Karvinen, P., Stadler, H.-C., Dinapoli, R., Bunk, O. \& Menzel, A. (2014). Opt. Express, 22, 14859-14870.

Johnson, I., Bergamaschi, A., Billich, H., Cartier, S., Dinapoli, R., Greiffenberg, D., Guizar-Sicairos, M., Henrich, B., Jungmann, J., Mezza, D., Mozzanica, A., Schmitt, B., Shi, X. \& Tinti, G. (2014). J. Instrum. 9, C05032.

Karim, W., Spreafico, C., Kleibert, A., Gobrecht, J., VandeVondele, J., Ekinci, Y. \& van Bokhoven, J. A. (2017). Nature (London), 541, 6871

Kleibert, A., Passig, J., Meiwes-Broer, K.-H., Getzlaff, M. \& Bansmann, J. (2007). J. Appl. Phys. 101, 114318. 
Kraft, P., Bergamaschi, A., Broennimann, Ch., Dinapoli, R., Eikenberry, E. F., Henrich, B., Johnson, I., Mozzanica, A., Schlepütz, C. M., Willmott, P. R. \& Schmitt, B. (2009). J. Synchrotron Rad. 16, 368-375.

Krajnak, M., McGrouther, D., Maneuski, D., Shea, V. O. \& McVitie, S. (2016). Ultramicroscopy, 165, 42-50.

Kuch, W., Gilles, J., Offi, F., Kang, S., Imada, S., Suga, S. \& Kirschner, J. (2001). Surf. Sci. 480, 153-162.

Kühlbrandt, W. (2014). Science, 343, 1443-1444.

Leanza, D., Vaz, C. A. F., Czekaj, I., Novák, P. \& Kazzi, M. E. (2017). Unpublished.

Le Guyader, L., Kleibert, A., Fraile Rodríguez, A., El Moussaoui, S., Balan, A., Buzzi, M., Raabe, J. \& Nolting, F. (2012). J. Electron Spectrosc. Relat. Phenom. 185, 371-380.

Llopart, X., Campbell, M., Dinapoli, R., San Segundo, D. \& Pernigotti, E. (2002). IEEE Trans. Nucl. Sci. 49, 2279-2283.

Locatelli, A. \& Bauer, E. (2008). J. Phys. Condens. Matter, 20, 093002.

Lu, A.-H., Salabas, E. L. \& Schüth, F. (2007). Angew. Chem. Int. Ed. 46, 1222-1244.

McMullan, G., Cattermole, D. M., Chen, S., Henderson, R., Llopart, X., Summerfield, C., Tlustos, L. \& Faruqi, A. R. (2007). Ultramicroscopy, 107, 401-413.

McMullan, G., Chen, S., Henderson, R. \& Faruqi, A. (2009). Ultramicroscopy, 109, 1126-1143.

Moldovan, G., Matheson, J., Derbyshire, G. \& Kirkland, A. (2008). Nucl. Instrum. Methods Phys. Res. A, 596, 402-408.

Nederlof, I., van Genderen, E., Li, Y.-W. \& Abrahams, J. P. (2013). Acta Cryst. D69, 1223-1230.

NIST (2017). Estar, http://physics.nist.gov/PhysRefData/Star/Text/ ESTAR.html.
Pratt, A., Lari, L., Hovorka, O., Shah, A., Woffinden, C., Tear, S. P., Binns, C. \& Kröger, R. (2014). Nat. Mater. 13, 26-30.

Quantum Detectors, (2017). Merlin for EM, http://quantumdetectors. com/merlin-for-em/.

Raighne, A. M., Fernandez, G. V., Maneuski, D., McGrouther, D. \& O' Shea, V. (2011). J. Instrum. 6, C01047.

Rossi, L., Fischer, P., Rohe, T. \& Wermes, N. (2006). Pixel Detectors, From Fundamentals to Applications. Berlin: Springer-Verlag.

Schlier, R. E. \& Farnsworth, H. E. (1959). J. Chem. Phys. 30, 917-926.

Schmidt, T., Marchetto, H., Lévesque, P. L., Groh, U., Maier, F., Preikszas, D., Hartel, P., Spehr, R., Lilienkamp, G., Engel, W., Fink, R., Bauer, E., Rose, H., Umbach, E. \& Freund, H.-J. (2010). Ultramicroscopy, 110, 1358-1361.

Schmidt, T., Sala, A., Marchetto, H., Umbach, E. \& Freund, H.-J. (2013). Ultramicroscopy, 126, 23-32.

Schneider, C. M. (1997). J. Magn. Magn. Mater. 175, 160-176.

Sikharulidze, I., van Gastel, R., Schramm, S., Abrahams, J., Poelsema, B., Tromp, R. \& van der Molen, S. (2011). Nucl. Instrum. Methods Phys. Res. A, 633, S239-S242.

Sobott, B. A., Broennimann, Ch., Schmitt, B., Trueb, P., Schneebeli, M., Lee, V., Peake, D. J., Elbracht-Leong, S., Schubert, A., Kirby, N., Boland, M. J., Chantler, C. T., Barnea, Z. \& Rassool, R. P. (2013). J. Synchrotron Rad. 20, 347-354.

Stöhr, J. (1995). J. Electron Spectrosc. Relat. Phenom. 75, 253.

Tinti, G., Bergamaschi, A., Cartier, S., Dinapoli, R., Greiffenberg, D., Johnson, I., Jungmann-Smith, J. H., Mezza, D., Mozzanica, A., Schmitt, B. \& Shi, X. (2015). J. Instrum. 10, C03011.

Vaz, C. A. F., Balan, A., Nolting, F. \& Kleibert, A. (2014). Phys. Chem. Chem. Phys. 16, 26624-26630.

Veneklasen, L. H. (1991). Ultramicroscopy, 36, 76-90. 